\title{
Reward systems, cognition, and emotion: Introduction to the special issue
}

\author{
Marie T. Banich ${ }^{1} \cdot$ Stan Floresco ${ }^{2}$ \\ Published online: 23 May 2019 \\ (C) The Psychonomic Society, Inc. 2019
}

Within the past decade or so, our knowledge regarding reward systems in the brain, the nature of their interactions with other brain systems, and their influence on a wide range of cognitive and emotional processes has grown substantially. Interest in these topics is growing because they are enriching knowledge from the viewpoint of basic science and also because of their implications for expanding our understanding of various psychiatric and neurological disorders. Building on the tradition of CABN to report research that allows for linkages between psychological theory, neural mechanisms, and computational approaches (Banich, 2016), we have assembled a set of 21 papers that provide a broad domain of perspectives on critical and emerging issues in the field. This breadth is reflected in the range methodologies employed across the papers, from studies with animal models to human participants, from neurologically normal individuals to those who have suffered brain damage, and using methods that probe neural and cognitive processes ranging from fMRI to ERPs to pupillometry. Breadth is also provided by theoretical reviews that synthesize accumulated knowledge and present it within a new and integrated framework. Although the papers in the special issue cover a broad series of topics, themes nonetheless emerge, which provide insight into the critical issues facing the field today. In each of the following sections, we discuss those issues and the perspective provided by the contributions to this special issue.

Marie T. Banich

marie.banich@colorado.edu

Stan Floresco

floresco@psych.ubc.ca

1 Institute of Cognitive Science, Department of Psychology and Neuroscience, University of Colorado Boulder, Boulder, CO, USA

2 Department of Psychology, University of British Columbia, Vancouver, British Columbia, Canada

\section{Neurotransmitter systems related to reward}

The first two papers in this special issue consider the role that specific neurotransmitters play in the brain's reward circuity. When one thinks of a neurotransmitter system that plays a critical role in reward processing, the dopaminergic system is the one that inevitably pops to mind. Its role has been examined in regard to reinforcement learning, as dopamine is thought to aid in reward-related and error-driven learning (Schultz, Dayan, \& Montague, 1997). In addition, disruptions and "hijacking" of the reward systems have been implicated in addiction (Volkow, Wise, \& Baler, 2017). In the first paper of this section, Berry et al. (2019, this issue) focus on how dopaminergic systems influence decision-making from the perspective of aging. Dopamine levels are known to decline with age (Karrer, Josef, Mata, Morris, \& Samanez-Larkin, 2017), and Berry and colleagues consider the implications of this phenomenon for alterations in decision-making with age, as well as individual differences in the nature of this decline. They also discuss the degree and conditions under which the well-documented affective bias in older adults toward positive material (Mather, 2016) may counteract decision-making processes that could be compromised by reduced levels of dopamine.

The second paper in this special issue reviews an entirely different neurotransmitter (and peptide) system, the opioid system. This system is known to reduce pain; in fact, relief from noxious or painful stimuli may also be perceived as rewarding, as well as increasing the "liking" of stimuli. In their contribution, van Steenbergen et al. (2019, this issue) review how the opioid system influences motivation and cognitive control. In particular, they suggest that the opioid system influences similar brain regions to that of the dopaminergic system, including orbitofrontal cortex, basal ganglia, amygdala, anterior cingulate cortex, and prefrontal cortex. They argue that the opioid system's mechanisms likely influence decision-making and cognitive control by increasing the subjective value of reward and reducing aversive arousal. 


\section{Value-based decision-making and reinforcement learning}

Some papers in this special issue examine value-based decision-making - that is, how we choose between options that differ in their rewards and costs. In their paper, Orsini et al. (2019, this issue) provide a conceptual framework from which to consider this question and demonstrate how optogenetic techniques in animals can be used to provide unique insights. In particular, they emphasize how neural activity occurring at distinct points of the decision process can differentially influence choice. Providing insights from studies of individuals with circumscribed lesions, Spaniol et al. (2019, this issue) evaluated the decision-making abilities of patients with ventromedial prefrontal (vmPFC) lesions compared with patients with lesions in other regions and neurologically intact controls on a dynamic decision-making task. From their investigation, they conclude that the vmPFC is particularly important for adaptive decision-making under conditions that are affect rich as compared with decisions without an affective component. Another paper by Fontanesi et al. (2019, this issue) used computational approaches to unravel the relationship between choice preferences and reaction times during reinforcement learning. Studies of perceptual decision-making have shown a clear relationship between these variables, but how they may relate during reward-related decision-making is less clear. The authors showed that the valence (positive vs. negative) of the potential outcome primarily affected decision speed, whereas the relative clarity of the feedback after decisions influenced both reaction times and decision accuracy. These findings highlighted the subtle differences in the mechanisms that may mediate decisions occurring in different contexts.

\section{The interface between reward/motivation and memory}

One of the seminal ideas with regard to reward processing, as mentioned above, is that dopamine serves as a signal to support error-driven learning. A major site in the brain in which such computations are thought to occur is the basal ganglia (see review by Lau, Monteiro, \& Paton, 2017), which is implicated in the procedural/implicit learning system. This system is posited to allow for learning that is incremental and slow, occurring via statistical regularities across different similar experiences so as to yield overlapping representations of events. However, another brain system, the hippocampus and associated midline structures, is also thought to play an important role in memory and learning. In contrast to the basal ganglia, these brain structures allow for fast learning from a specific instance, enabling information learned in differing contexts to be linked to one another, and provides a means for distinct and specific learning events to be clearly discriminated (O'Reilly \& Norman, 2002). Two papers in this issue consider how functioning of this latter system, the hippocampal system, is related to reward processing.

Using fMRI, Frank et al. (2019, this issue) show that reward-related information influences this hippocampal system via its functional connectivity with reward-related regions, including the ventral striatum, orbitofrontal cortex and the anterior cingulate during performance of an episodic memory task. In their task, a precue indicated the amount of reward that could be obtained for remembering a subsequently presented object pair. Hence, connectivity serves as the glue to bind specific instances to their reward-related attributes. Furthermore, they demonstrated that differences among individuals in how much memory performance was modulated by reward is associated with connectivity between the hippocampus and this set of reward-related regions. Palombo et al. (2019, this issue) demonstrate the role of the hippocampus in value-based learning using converging evidence from brain activation observed via fMRI and from the examination of patients with hippocampal damage. In their probabilistic learning task, participants saw "players" in a game and had to guess whether that player would win money or not. In addition to ventral striatal regions, fMRI revealed that this task activated the hippocampus. Although neuroimaging suggested hippocampal involvement, an important question is whether the hippocampus is necessary for such learning, or is just coactivated as a minor player along with structures that may play a more major role. Providing evidence for a critical role of the hippocampus in such learning, they observed that seven patients with hippocampal lesions were impaired at the task.

\section{How does reward influence attention and cognitive control?}

In a parallel manner to considering how reward might influence memory and value-based learning, another set of papers examines how reward influences other cognitive functions, including attention and/or cognitive control. Walsh et al. (2019, this issue) show that reward for accurate performance in a simple letter discrimination task helps to attenuate the effects of irrelevant distracting information, even when it is emotional in nature, suggesting that reward can act to focus attention. Of note, they used pupillometry to provide insights into how such focusing of attention may occur. In particular, they found that reward altered the time course of control such that there was a quick shift in pupil dilation preceding stimulus presentation, suggesting a proactive process to prepare to disengage from distractors.

In a somewhat related manner, Asci et al. (2019, this issue) examined the conditions under which reward engenders the 
need for cognitive control. In their task, individuals made a simple judgement with regard to a number's value (less than 5 , greater than 5), with one category being mapped onto a Go response and the other to a No-Go response, each of which were equiprobable. The color of the number indicated whether or not it was possible to receive a reward in the form of a monetary bonus. Reward augmented action tendencies leading to faster responding on Go trials and more difficulty in withholding a response (commission errors) on No-Go trials. Of interest was the degree to which these patterns were associated with activation in cognitive control regions. Activation was higher in prefrontal cognitive control regions (i.e., DLPFC) during nonrewarded as compared with rewarded Go trials, but increased conversely during rewarded as compared to nonrewarded No-Go trials. These findings suggest that action-related biases promoted by reward may, under the appropriate circumstances, induce or require cognitive control.

The next paper, that of Orr et al. (2019, this issue), examined the degree to which reward influences processing at a higher level of cognitive control - that of choosing which task or goal should be selected. In their task, the overall goal was to choose randomly, across trials, which of two tasks to perform. One task involved deciding which of two numerals was presented in a larger font, and the other involved deciding which of two numerals had a higher value. Rewards were delivered probabilistically and relatively infrequently (25\%) across those trials on which a correct response was made. While the receipt of a reward did not influence task choice on the subsequent trial, it was associated with increased activation in reward-related brain regions. In addition, during the period of task choice at the beginning of the subsequent trial, increased activity was observed in brain regions, such as the rostrolateral prefrontal cortex, that are involved in cognitive control and higher order goal selection. This finding suggests integration of reward-related information with brain systems that choose and support goal-driven processing.

\section{How do attention, cognitive control, motivation, or effort influence reward processing?}

While the papers above examine how reward influences different processes such as learning and cognitive control, one can also examine the reverse question - that is, "What processes and influences modify the value or effect of reward?" The work by Nadig et al. (2019, this issue) demonstrates how the scope of attention can influence the effect of rewards. They had individuals perform a monetary incentive delay task, a paradigm in which individuals see a cue indicating whether they can (or cannot) receive a reward on the current trial if they respond within a specified response deadline to a target object (e.g., white square). Here, they altered the paradigm to have the target object be a Navon figure, in which letters small in size (e.g., $H$ s, $S$ s) are arranged to form a larger letter (e.g., an $H$, an $S$ ). In one condition, attention had to be directed more locally and focally to the small letter, while in the other condition attention was dispersed more globally to the large letter. Directing attention to the local letters, in a more focal manner, led to fewer errors and better performance, as reflected by larger earnings. In addition, when an incentive was present (i.e., there was an opportunity to win money), a narrow attentional scope was associated with a larger physiological response (i.e., a Feedback P3 ERP) to negative feedback, whereas a broader attentional scope was associated with an increased ERP response to positive feedback. These results show how attentional scope can influence differential responses to positive as compared with negative feedback.

In a review of the literature spanning behavioral studies, fMRI and EEG/ERPs, Kelley et al. (2019, this issue) argue that self-control, which they define as the ability to override or alter motivated responses, may actually increase rather than decrease subsequent reward processing. Instead of conceptualizing resources as being reduced by self-control, they review the literature to argue that because control is effortful and demands resources, it induces negative affect, which, then in an opponent-process manner, causes an individual to seek reward. This seeking of reward is reflected in shifts in attention-related and valuation processes toward rewards. Hence, implementing self-control counterintuitively ends up making rewards more enticing. They review evidence for this proposition from studies across various domains ranging from food desire and consumption to substances of abuse to monetary incentives and rewards.

Two other articles in the issue consider the relationship of motivation or effort with regard to reward processing and the interface of these processes with cognitive control. To examine the influence of reward and effort separately, Vassena et al. (2019, this issue) designed a task that orthogonally varied the degree of possible reward as well as the degree of effort required to perform an arithmetic addition task. In the first experiment, participants were cued serially as to the degree of effort required and subsequently the degree of reward available (or vice versa) prior to trial onset. Presenting reward information first led to increased accuracy, especially when the reward was large. The second experiment was similar, except that now individuals decided whether to perform the task as stipulated by the cues presented on a given trial, or default to an easy/low reward trial. Under these conditions, presenting reward information first blunted the effect of reward on the decision-making process. They then go on to show how this seemingly counterintuitive pattern of results can be predicted by a computational model that formalizes the manner in which prefrontal regions engage in error prediction so as to exert cognitive control. 
Using an individual differences approach, Damme and colleagues (2019, this issue) link issues of cognitive control and reward from the perspective of motivation - that is, the willingness to work for a reward. Their study examined the degree to which levels of executive functioning are linked to willingness to work for a reward. Those individuals higher in executive functioning were more willing to work for a reward when it was likely, but not when such efforts were unlikely, to lead to a reward, suggesting a conservation of effort. Moreover, these researchers demonstrate that the relationship between an individual's level of functioning with regard to willingness to work for a reward is independent from the willingness to delay receiving a reward. This work, along with the other papers mentioned above, indicate that numerous factors can modulate a reward's value, as well as the effort exerted to obtain it.

\section{Developmental and life-span perspectives}

Some papers in this issue examine the interaction between reward-based processing and cognitive control from a developmental perspective. One of the major conceptual models of adolescent brain development posits a mismatch between an overly active reward system, whose activity peaks in midadolescence, and an underdeveloped and still maturing cognitive control system. It has been hypothesized that this "mismatch" can help to explain many of the risk-taking behaviors observed in adolescents (Casey, 2015; Steinberg, 2010). Consistent with such a viewpoint is the work by McKewen et al. (2019, this issue), who found evidence for increasing cognitive control and control over decision-making processes in a sample of 15-to-35-year-olds. They observed that a self-reported cognitive control system acts as a mediator between impulsivity and psychological distress, but such a relationship was observed across their sample regardless of age and was not specific to adolescents. With the papers discussed below, this research implicates individuals' differences as being important to consider with regard to reward and reward processing.

In a related vein, Maresh and colleagues (2019, this issue) examine the impact of reward and control systems in adolescents with and without bipolar disorder on a monetary incentive delay task. Their research used frontal theta as an index of cognitive control and frontal alpha asymmetry as an index of motivational approach tendencies. They observed that with increasing age, healthy individuals engaged less cognitive control during anticipation of reward on trials with negative as compared with positive motivational value. Such an effect was not observed for adolescents with bipolar disorder, suggesting less differentiation in cognitive control as a function of the valence of motivational cues. In addition, they found a gender differences in that healthy adolescent girls has less of a need to engage cognitive control mechanisms, as indexed by reduced activity in the theta band recorded over frontal regions, to reach the same level of performance than did girls with bipolar disorder, boys with bipolar disorder, and healthy boys. This finding demonstrates the interplay in adolescence between cognitive control with regard to reward, approach, and motivational systems. The findings also highlight the degree to which it is important to consider the influence of gender on reward processing, at least during this adolescent time period.

At the other end of the developmental spectrum Yee et al. (2019, this issue) examine how motivational incentives influence the performance of younger versus older adults on a cognitive control task, a consonant-voxel/odd-even taskswitching paradigm. At the beginning of each trial, individuals were shown the degree of money, a secondary incentive, they could obtain for a correct performance. After a correct performance on a trial, they received both the monetary reward of the size indicated by the cue as well as receipt of a primary incentive, a liquid, that was either positive (apple juice), neutral (isotonic tasteless solution), or negative (seawater). Older adults' performance on this cognitive control task could be increased by incentive motivation cues, so much so that they matched performance of younger adults, although their learning was more incremental and required more practice. However, the processing of motivational cues by older adults was qualitatively different. Over time, younger adults could integrate information between two types of incentives (liquid and money), whereas older adults tend to be influenced by one or the other. This work provides new insights into the types of subtle but important effects reward may have on cognitive control with aging. Another perspective on age effects with reward is the review paper by Berry and colleagues (2019, this issue), discussed above, that focuses on the degree to which changes in dopaminergic function, especially in aging, can influence decision-making.

\section{Individual differences in neural organization and function related to reward processing}

In addition to the effects of development status, three other papers in this special issue consider the influence of how differences in the architecture of the nervous system and brain might influence reward processing. One paper examines this issue from the perspective of genetic variation putatively associated with norepinephrine availability. Ehlers et al. (2019, this issue) find that what is viewed as rewarding is influenced by genetic variation putatively associated with norepinephrine availability. It had been shown previously that individuals with greater norepinephrine availability have a greater attentional bias toward and more vivid perceptual perception of emotionally salient stimuli. Here, they examine whether this 
variation influences the perception of ambiguous facial stimuli. Individuals were initially asked to categorize a series of faces that included happy and angry faces as well as ambiguous morphs between these two categories, and then again after exposure to a series of angry faces. This latter exposure induces a bias toward viewing ambiguous faces as happier (and by inference more rewarding), a bias that was increased in individuals with greater norepinephrine availability.

The two other papers related to this theme show that connectivity between brain regions influences reward processing. Adrián-Ventura et al. (2019, this issue) find that individuals higher in reward sensitivity show increased connectivity at rest between brain regions that are likely portions of the mesocortical dopaminergic system. In particular, they found that individuals higher in reward sensitivity show greater connectivity between the ventral tegmental areas and the ventromedial prefrontal cortex, often implicated in valuations, as well as between the ventromedial prefrontal cortex and the anterior cingulate, which has been found to be involved in the degree of effort exerted for a reward (see other papers in this issue on the effort expended for a reward: Damme et al. 2019; Vasenna et al.). This paper highlights the degree to which reward is processed in the brain by an integrated system spanning many regions with various putative functions. Also as discussed earlier, Frank et al. (2019, this issue) demonstrated that individual differences in connectivity between the hippocampus and some of the same regions implicated by Adrián-Ventura and colleagues, including the anterior cingulate cortex and orbitofrontal cortex, influences the degree to which performance on a paired-associate memory task is influenced by potential reward.

\section{Implications for psychopathology}

The final subgroup of papers within this issue examines how dysfunction in reward systems and/or their interactions with cognitive systems may contribute to symptoms associated with certain psychiatric and behavioral disorders. In their paper, Hellberg et al. (2019, this issue) review findings suggesting that some of the pathophysiological mechanisms that contribute to gambling disorder may be related to those that underlie certain aspects of drug addiction. A prominent theory posits that drugs of abuse may sensitize the dopamine system, which in turn enhances sensitivity to drug-related cues to enhance drug craving (Robinson \& Berridge, 2008). These authors note that the uncertainty associated with games of chance may induce similar changes in dopamine signaling in susceptible individuals that may underlie gambling addiction.

Gheza et al. (2019, this issue) examine reinforcement learning in a large cohort of individuals with and without major depressive disorder. One of the emerging themes from research on individuals with depression is the suggestion that these individual show abnormalities in reward processing, especially in the context of stress (e.g., Pizzagalli, 2014). Here, Gheza et al. administered a standard reinforcement learning task and used frontal-midline theta power time-locked to the response and feedback as neurophysiological measures of processing. Similar to the work of Maresh et al. (2019, this issue), they used the asymmetry of frontal alpha as a measure of approachrelated motivation. Behaviorally, the individuals with major depressive disorder showed no deficits on the rewardlearning task compared with controls, and for the most part, their frontal-midline theta power did not vary, except when reward probability was low. What seemed to distinguish the groups, however, reduced approach-related motivation, as indexed by alpha asymmetry, in the depressive group during the task execution. This pattern of results suggests that while individuals with major depressive disorder can learn from reinforcement and reward, they may have reduced motivation to do so. Another paper examining issues related to psychopathology, discussed above, is that of Maresh et al. (2019, this issue), which also showed an effect of motivation-in this case, motivational cues. They observed that adolescents with bipolar disorder did not show increasing differentiation of cognitive control as a function of the valence of motivational cues with increasing age, as was observed for healthy control individuals.

In sum, the papers contained in this special issue provide both diverse and sometimes convergent perspectives on the many different ways in which reward processing intersects with cognitive, motivational, and emotional processes, and the neurobiological and computational mechanisms that undergird those interactions.

\section{References}

Adrián-Ventura, J., Costumero, V., Parcet, M.A., \& Ávila, C. (2019). Reward network connectivity "at rest" is associated with reward sensitivity in healthy adults: A resting-state fMRI study. Cognitive, Affective, \& Behavioral Neuroscience.https://doi.org/10.3758/ s13415-019-00688-1

Asci, O., Braem, S., Park, H. R. P., Boehler, C. N., \& Krebs, R. M. (2019). Neural correlates of reward-related response tendencies in an equiprobable go/no-go task. Cognitive, Affective, \& Behavioral Neurosciencehttps://doi.org/10.3758/s13415-019-00692-5

Banich, M. T. (2016). The future of $\mathrm{CABN}-\mathrm{A}$ conceptual triad: Psychological theory, neurobiological approaches, computational methods. Cognitive, Affective, \& Behavioral Neuroscience, 16(1), 1-2. https://doi.org/10.3758/s13415-016-0401-z

Berry, A. S., Jagust, W. J., \& Hsu, M. (2019). Age-related variability in decision-making: Insights from neurochemistry. Cognitive, Affective, \& Behavioral Neuroscience. https://doi.org/10.3758/ s13415-018-00678-9 
Casey, B. J. (2015). Beyond simple models of self-control to circuit-based accounts of adolescent behavior. Annual Review of Psychology, 66, 295-319. https://doi.org/10.1146/annurev-psych-010814-015156

Damme, K. S. F., Kelley, N. J., Quinn, M. E., Glazer, J. E., Chat, I. K.-Y., Young, K. S., ... Craske, M. G. (2019). Emotional content impacts how executive function ability relates to willingness to wait and to work for reward. Cognitive, Affective, \& Behavioral Neuroscience. https://doi.org/10.3758/s13415-019-00712-4

Ehlers, M. R., Ross, C. J. D., \& Todd, R. M. (2019). The influence of the noradrenergic/stress system on perceptual biases for reward. Cognitive, Affective, \& Behavioral Neuroscience. https://doi.org/ 10.3758/s13415-018-00657-0

Fontanesi, L., Palminteri, S., \& Lebreton, M. (2019). Decomposing the effects of context valence and feedback information on speed and accuracy during reinforcement learning: A meta-analytical approach using diffusion decision modeling. Cognitive, Affective, \& Behavioral Neuroscience.

Frank, L. E., Preston, A. R., \& Zeithamova, D. (2019). Functional connectivity between memory and reward centers across task and rest track memory sensitivity to reward. Cognitive, Affective, \& Behavioral Neuroscience. https://doi.org/10.3758/s13415-01900700-8

Gheza, D., Bakic, J., Baeken, C., De Raedt, R., \& Pourtois, G. (2019). Abnormal approach-related motivation but spared reinforcement learning in MDD: Evidence from fronto-midline theta oscillations and frontal alpha asymmetry. Cognitive, Affective, \& Behavioral Neuroscience. https://doi.org/10.3758/s13415-019-00693-4

Hellberg, S. N., Russell, T. I., \& Robinson, M. J. F. (2019). Cued for risk: Evidence for an incentive sensitization framework to explain the interplay between stress and anxiety, substance abuse, and reward uncertainty in disordered gambling behavior. Cognitive, Affective, \& Behavioral Neuroscience. https://doi.org/10.3758/s13415-01800662-3

Karrer, T. M., Josef, A. K., Mata, R., Morris, E. D., \& Samanez-Larkin, G. R. (2017). Reduced dopamine receptors and transporters but not synthesis capacity in normal aging adults: A meta-analysis. Neurobiology of Aging, 57, 36-46.

Kelley, N. J., Finley, A. J. \& Schmeichel, B. J. (2019). After-effects of self-control: The reward responsivity hypothesis. Cognitive, Affective, \& Behavioral Neuroscience. https://doi.org/10.3758/ s13415-019-00694-3

Lau, B., Monteiro, T., \& Paton, J. J. (2017). The many worlds hypothesis of dopamine prediction error: implications of a parallel circuit architecture in the basal ganglia. Current Opinion in Neurobiology, 46, 241-247. http://doi.org/10.1016/j.conb.2017.08.015. https://doi. org/10.1016/j.conb.2017.08.015

Maresh, E., Stim, J., Van Voorhis, A., Kang, S. S., Luciana, M., Sponheim, S., \& Urosevic, S. (2019). Neurophysiological correlates of cognitive control and approach motivation abnormalities in adolescent bipolar disorders. Cognitive, Affective, \& Behavioral Neuroscience.

Mather, M. (2016). The affective neuroscience of aging. Annual Review of Psychology, 67, 213-238. https://doi.org/10.1146/annurev-psych122414-033540

McKewen, M., Skippen, P., Cooper, P. (2019). Does cognitive control ability mediate the relationship between reward-related mechanisms, impulsivity and maladaptive outcomes in adolescence and young adulthood? Cognitive, Affective, \& Behavioral Neuroscience.

Nadig, A., Kelley, N. J., Pornpattananangkul, N., Glazer, J. E., \& Nusslock, R. (2019). Shifts in attentional scope modulate eventrelated potentials evoked by reward. Cognitive, Affective, \& Behavioral Neuroscience. https://doi.org/10.3758/s13415-01900705-3
O’Reilly, R., \& Norman, K. (2002). Hippocampal and neocortical contributions to memory: Advances in the complementary learning systems framework. Trends in Cognitive Sciences, 6(12), 505-510.

Orr, J. M., Imburgio, M. J., Bernard, J. A., \& Banich, M. T. (2019). Striatal-frontal network activation during voluntary task selection under conditions of monetary reward. Cognitive, Affective, \& Behavioral Neuroscience. https://doi.org/10.3758/s13415-01900689-0

Orsini, C. A., Hernandez, C. M., Bizon, J. L., \& Setlow, B. (2019). Deconstructing value-based decision making via temporally selective manipulation of neural activity: Insights from rodent models. Cognitive, Affective, \& Behavioral Neuroscience. https://doi.org/10. 3758/s13415-018-00649-0

Palombo, D. J., Hayes, S. M., Reid, A. G., \& Verfaellie, M. (2019). Hippocampal contributions to value-based learning: Converging evidence from fMRI and amnesia. Cognitive, Affective, \& Behavioral Neuroscience. https://doi.org/10.3758/s13415-018-00687-8

Pizzagalli, D. A. (2014). Depression, stress, and anhedonia: toward a synthesis and integrated model. Annual Review of Clinical Psychology, 10(1), 393-423. https://doi.org/10.1146/annurevclinpsy-050212-185606

Robinson, T. E., \& Berridge, K. C. (2008). The incentive sensitization theory of addiction: Some current issues. Philosophical Transactions of the Royal Society of London Series B, Biological Sciences, 363(1507), 3137-3146

Schultz, W., Dayan, P., \& Montague, P. R. (1997). A neural substrate of prediction and reward. Science, 275, 1593-1599.

Spaniol, J., Di Muro, F., \& Ciaramelli, E. (2019). Differential impact of ventromedial prefrontal cortex damage on "hot" and "cold" decisions under risk. Cognitive, Affective, \& Behavioral Neuroscience. https://doi.org/10.3758/s13415-018-00680-1

Steinberg, L. (2010). A dual systems model of adolescent risk-taking. Developmental Psychobiology, 52(3), 216-224. https://doi.org/10. 1002/dev.20445

van Steenbergen, H., Eikemo, M., \& Leknes, S. (2019). The role of the opioid system in decision making and cognitive control: A review. Cognitive, Affective, \& Behavioral Neuroscience. https://doi.org/10. 3758/s13415-019-00710-6

Vassena, E., Deraeve, J., \& Alexander, W. H. (2019). Task-specific prioritization of reward and effort information: Novel insights from behavior and computational modeling. Cognitive, Affective, \& Behavioral Neuroscience. https://doi.org/10.3758/s13415-01800685-w

Volkow, N. D., Wise, R. A., \& Baler, R. (2017). The dopamine motive system: Implications for drug and food addiction. Nature Reviews Neuroscience, 18(12), 741-752. https://doi.org/10.1038/nrn.2017. 130

Walsh, A. T., Carmel, D., \& Grimshaw, G. M. (2019). Reward elicits cognitive control over emotional distraction: Evidence from pupillometry. Cognitive, Affective, \& Behavioral Neuroscience. https://doi.org/10.3758/s13415-018-00669-w

Yee, D. M., Adams, S., Beck, A., \& Braver, T. S. (2019). Age-related differences in motivational integration and cognitive control. Cognitive, Affective, \& Behavioral Neuroscience. https://doi.org/ 10.3758/s13415-019-00713-3

Publisher's note Springer Nature remains neutral with regard to jurisdictional claims in published maps and institutional affiliations. 